\title{
Relative effectiveness of insulin pump treatment over multiple daily injections and structured education during flexible intensive insulin treatment for type 1 diabetes: cluster randomised trial (REPOSE)
}

\section{The REPOSE Study Group}

Correspondence to: $\mathrm{S}$ Heller

Department of Oncology and

Metabolism, University of

Sheffield, Sheffield, UK

s.heller@sheffield.ac.uk

Cite this as: BMJ 2017;356:j1285 http://dx.doi.org/10.1136/bmj.j1285

Accepted: 8 February 2017

\author{
ABSTRACT \\ OBJECTIVE \\ To compare the effectiveness of insulin pumps with \\ multiple daily injections for adults with type 1 \\ diabetes, with both groups receiving equivalent \\ training in flexible insulin treatment. \\ DESIGN \\ Pragmatic, multicentre, open label, parallel group, \\ cluster randomised controlled trial (Relative \\ Effectiveness of Pumps Over MDI and Structured \\ Education (REPOSE) trial).
}

SETTING

Eight secondary care centres in England and Scotland. PARTICIPANTS

Adults with type 1 diabetes who were willing to undertake intensive insulin treatment, with no preference for pumps or multiple daily injections. Participants were allocated a place on established group training courses that taught flexible intensive insulin treatment ("dose adjustment for normal eating," DAFNE). The course groups (the clusters) were then randomly allocated in pairs to either pump or multiple daily injections.

\section{INTERVENTIONS}

Participants attended training in flexible insulin treatment (using insulin analogues) structured around the use of pump or injections, followed for two years.

\section{MAIN OUTCOME MEASURES}

The primary outcomes were a change in glycated haemoglobin (HbA1c) values (\%) at two years in participants with baseline $\mathrm{HbA} 1 \mathrm{c}$ value of $\geq 7.5 \%$ (58 $\mathrm{mmol} / \mathrm{mol}$ ), and the proportion of participants

\section{WHAT IS ALREADY KNOWN ON THIS TOPIC}

Assessments of insulin pump treatment in type 1 diabetes concluded that it was worthwhile for those who were otherwise unable to achieve good glycaemic control without disabling hypoglycaemia

The case for wider use is uncertain, given the small size and short duration of trials of pumps versus modern multiple daily injections, and the need to distinguish the effects of the pump and the extra education provided

\section{WHAT THIS STUDY ADDS}

The REPOSE trial randomised patients to pump or multiple daily injections, with both groups receiving similar structured education; $\mathrm{HbA1c}$ levels and rates of severe hypoglycaemia decreased in both groups, slightly more in the pump group, but without statistical significance

Both groups showed improved quality of life benefits, but those using pumps showed additional albeit modest benefits in quality of life, reported fewer restrictions in diet and daily hassles in the diabetes specific quality of life scale, and greater treatment satisfaction

Although participation in the courses produced sustained improvement, levels of glucose control remained far short of those currently recommended by NICE achieving an $\mathrm{HbA} 1 \mathrm{c}$ value of $<7.5 \%$. Secondary outcomes included body weight, insulin dose, and episodes of moderate and severe hypoglycaemia. Ancillary outcomes included quality of life and treatment satisfaction.

\section{RESULTS}

317 participants ( 46 courses) were randomised (156 pump and 161 injections). 267 attended courses and 260 were included in the intention to treat analysis, of which 235 (119 pump and 116 injection) had baseline $\mathrm{HbA1c}$ values of $\geq 7.5 \%$. Glycaemic control and rates of severe hypoglycaemia improved in both groups. The mean change in $\mathrm{HbA} 1 \mathrm{c}$ at two years was $-0.85 \%$ with pump treatment and $-0.42 \%$ with multiple daily injections. Adjusting for course, centre, age, sex, and accounting for missing values, the difference was $-0.24 \%(-2.7 \mathrm{mmol} / \mathrm{mol})$ in favour of pump users ( $95 \%$ confidence interval -0.53 to 0.05 , $\mathrm{P}=0.10)$. Most psychosocial measures showed no difference, but pump users showed greater improvement in treatment satisfaction and some quality of life domains (dietary freedom and daily hassle) at 12 and 24 months.

\section{CONCLUSIONS}

Both groups showed clinically relevant and long lasting decreases in $\mathrm{HbA1c}$, rates of severe hypoglycaemia, and improved psychological measures, although few participants achieved glucose levels currently recommended by national and international guidelines. Adding pump treatment to structured training in flexible intensive insulin treatment did not substantially enhance educational benefits on glycaemic control, hypoglycaemia, or psychosocial outcomes in adults with type 1 diabetes. These results do not support a policy of providing insulin pumps to adults with poor glycaemic control until the effects of training on participants' level of engagement in intensive self management have been determined.

\section{TRIAL REGISTRATION}

Current Controlled Trials ISRCTN61215213.

\section{Introduction}

People with type 1 diabetes mellitus require lifelong treatment with insulin to prevent diabetic ketoacidosis and to optimise blood glucose levels to minimise vascular complications. ${ }^{1}$ Insulin is generally administered by multiple daily subcutaneous injections, using different insulins to cover background and meal requirements. Doses are adjusted according to eating, physical activity, and blood glucose level. This approach and its integration within flexible lifestyles is promoted in "dose adjustment for normal eating" (DAFNE) ${ }^{2}$ and similar structured training courses. ${ }^{3}$ Despite this training and 
best efforts, many people struggle to achieve glycaemic targets and a considerable proportion go on to develop serious complications, reducing both the length and the quality of their lives. ${ }^{14}$

In continuous subcutaneous insulin infusion, a pump delivers insulin continuously under the skin through a small plastic tube and cannula. ${ }^{56}$ Pumps are filled with quick acting insulin to supply both background insulin and insulin replacement after meals.

Potential advantages include more precise insulin delivery and the ability to adjust basal insulin levels. Observational studies have reported improved glucose control, reduced risk of hypoglycaemia, and enhanced quality of life. Pump treatment is more expensive than multiple daily injections, with pumps costing around $£ 2500$ ( \$3041; €2800) each plus $€ 1500$ a year for consumables (cannulas, reservoirs, and batteries). ${ }^{7}$

In the UK, pump use is approved in adults with type 1 diabetes who have high glycated haemoglobin (HbA1c) values $(>8.5 \%)$ or an inability to achieve reasonable control without "disabling hypoglycaemia." ${ }^{\circ}$ An estimated $6 \%$ of UK adults with type 1 diabetes use pumps, which is lower than in many comparable countries. ${ }^{9}$ Around $40 \%$ of people with type 1 diabetes in the USA use pumps, ${ }^{10}$ and proponents of pumps suggest that far more people should be offered them in the UK. ${ }^{11}$

One weakness of existing evidence is that patients allocated to pumps are likely to have received more training and attention than those using multiple daily injections. A recent observational study ${ }^{12}$ of pump treatment and injections, where both groups received intensive education in insulin usage, concluded that the training might have made the most difference. To our knowledge, no randomised trials in adults have compared pump treatment with multiple daily injections where the same structured training in insulin adjustment has been provided, so the added benefit of pump technology remains unclear.

In the Relative Effectiveness of Pumps Over MDI and Structured Education (REPOSE) trial we assessed the effectiveness of adding pump treatment to high quality equivalent structured education in flexible intensive insulin treatment for people with type 1 diabetes, comparing pump plus education with multiple daily injections plus education. Our hypothesis was that much of the benefit of pump treatment might come from the re-training and education in insulin use given to enable patients to use pumps safely. We present the clinical effectiveness and quantitative psychosocial results of this pragmatic trial.

\section{Methods}

Trial design

The study protocol has been previously published. ${ }^{13}$ In brief, we conducted a multicentre, parallel group, open label, confirmatory, cluster randomised controlled trial. Eight secondary care centres (three in Scotland and five in England) recruited up to 40 participants to three pump and three multiple daily injection courses (with five to eight patients on each course) over 11 months. Participants were allocated a place on a one week
DAFNE skills training course, with a further visit at six weeks. The course groups (clusters) were randomly allocated in pairs to either pump or multiple daily injections. After the courses, participants received the trial treatment for two years. We collected outcome measures at baseline (up to three weeks before the DAFNE course) and at six, 12, and 24 months. A cluster design was chosen to address the challenge of randomising participants and then finding suitable times for their attendance on a course of the correct allocation. We believe this approach reduced both recruitment bias and attrition rates before the course.

\section{Participants}

We recruited adults with a diagnosis of type 1 diabetes for at least 12 months, and who were willing to undertake intensive insulin treatment with self monitoring of blood glucose levels, counting of carbohydrate intake, and insulin self adjustment, with no preference for either pump or multiple daily injections. Participants were those with clinical indications for structured education in insulin treatment to optimise diabetes control who had not participated previously in structured training. We excluded those with a strong desire for pump treatment, those already using optimised multiple daily injections and meeting the criteria of the National Institute for Health and Care Excellence for pump treatment, those needing a pump in the opinion of the investigator, those with serious diabetic complications, and those unable to communicate in English. Courses (clusters) required between five and eight participants to maintain optimal course dynamics.

\section{Interventions}

Participants using multiple daily injections attended standard DAFNE structured education courses, ${ }^{2}$ which were conducted over five consecutive days and delivered to groups of five to eight adults as outpatients. The participants took insulin aspart, a quick acting insulin analogue, for meals, and twice daily injections of insulin detemir for background replacement. They used the Accu-Chek Aviva Expert Bolus Advisor System (Roche Diagnostics Ltd, Burgess Hill, UK) as a bolus calculator, as there is evidence that bolus advisers can improve glycaemic control, presumably by helping patients calculate the appropriate meal related bolus. ${ }^{14}$

Participants allocated to pump treatment attended a modified DAFNE course, previously validated in pump users. ${ }^{15}$ That course maintained the five day structure and the principles of insulin dose adjustment in the standard DAFNE course, but also incorporated the practical skills and learning outcomes needed to use pumps successfully. This necessitated an additional group session, delivered one to three weeks before the main course. Standard DAFNE includes a rigorous quality assurance programme. For the pump courses, fidelity testing was undertaken to assess incorporation of appropriate pump training. Participants used a Minimed Paradigm Veo insulin pump (model X54; Medtronic, Watford, UK) with insulin aspart. The bolus wizard in the pumps was activated as part of the course. 
The curriculum (and associated patient workbook) was adapted specifically to make better use of pump features, compared with standard (multiple daily injections) DAFNE. This included general pump management (infusion sets, cannulas, filling reservoirs, changing batteries, and troubleshooting). During the five day course participants were also taught use of the bolus wizard, basal testing and adjustment (including fasting during the day and overnight glucose profiles), use of temporary basal rates for physical activity or alcohol intake (reduced) and illness (increased), use of alternative bolus "waves" (extended, multiwave), prevention of diabetic ketoacidosis ("sick day rules" eg, when to use a pen to inject insulin in case of cannula/ pump failure, how much insulin to give and how often).

All participants (multiple daily injections and continuous subcutaneous insulin infusion) were invited to the course follow-up session at six to eight weeks, and those who required additional input were offered further one-to-one appointments. These appointments were supported by meter or pump downloads (Diasend: www.glooko.com/diasend; CareLink: www.medtronicdiabetes.com/products/carelink-personal-diabetes-software), depending on local availability. Participants were encouraged to maintain paper record diaries to facilitate discussion and adjustments, according to the principles taught on the course and supported by the workbook. Some might have already sought additional help in between these planned appointments. We recorded diabetes related contact with educators outside the course and at the six week follow-up.

\section{Outcomes}

\section{Primary outcomes}

We specified two primary outcomes. One was the change in HbA1c (\%, measured centrally) after two years in participants whose baseline $\mathrm{HbA1c}$ was $\geq 7.5 \%$ ( $58 \mathrm{mmol} / \mathrm{mol}$ ). HbA1c is the accepted ideal surrogate measure of glycaemic control and provides a measure of efficacy and a means of modelling long term cost effectiveness. Our choice of this primary outcome was based on our concern that HbA1c values might not decrease in those who entered the trial with low baseline values, but who might be experiencing problematic hypoglycaemia. Success for such individuals would be an HbA1c value that was maintained or even increased but with a reduced frequency of severe hypoglycaemia (an important secondary endpoint).

The other primary endpoint was the proportion of participants reaching the 2004 NICE target of HbA1c $\leq 7.5 \%(58 \mathrm{mmol} / \mathrm{mol})$.

\section{Secondary outcomes}

Secondary biomedical outcomes measured at six, 12, and 24 months were moderate hypoglycaemia (an episode that could be treated by the individual, but where hypoglycaemia caused a significant interruption of current activity leading to impaired performance, or embarrassment, or being woken during nocturnal sleep); severe hypoglycaemia (an episode leading to cognitive impairment sufficient to cause either coma or requiring the assistance of another person to recover); total and high density lipoprotein cholesterol levels; proteinuria; insulin dose; and body weight. Diabetic ketoacidosis was recorded through the assessment of serious adverse events throughout the trial.

\section{Ancillary outcomes}

Quantitative psychosocial self completed questionnaires assessed generic and diabetes specific quality of life: SF-12 (12 item short form health survey); WHOQOL-BREF (World Health Organization quality of lifeBREF); EQ-5D (EuroQol five dimensions questionnaire); DSQOL (diabetes specific quality of life), fear of hypoglycaemia (HFS: hypoglycaemia fear scale), satisfaction with treatment (DTSQ: diabetes treatment satisfaction questionnaire), and emotional wellbeing (HADS: hospital anxiety and depression scale) at the same time points. ${ }^{13}$

A health economic evaluation to address the question "What is the cost effectiveness of pump treatment compared with multiple daily injections in patients receiving the DAFNE structured education programme?" was undertaken. It has been submitted for publication. It included a within trial and a modelled patient lifetime analysis, the latter being the primary focus of the evaluation.

\section{Sample size}

We calculated the sample size using a minimally clinically important difference of $0.5 \%(5.5 \mathrm{mmol} / \mathrm{mol})$ in HbA1c values. To detect this difference with a standard deviation of $1 \%$ at $80 \%$ power and $5 \%$ two sided significance required 64 participants with an $\mathrm{HbA1c}$ of $\geq 7.5 \%$ in each group. To allow for clustering of educators, an average of seven participants per group, a within course intraclass correlation coefficient of 0.05 , and a $10 \%$ dropout rate, we required a sample size of 93 in each group. In the DAFNE database, ${ }^{15} 75 \%$ of participants had an $\mathrm{HbA1C}$ value $\geq 7.5 \%$, so we required 124 participants per group. We planned to recruit 280 participants, which increased the power to $85 \%$ but allowed for some variation in dropout rates and the proportion of participants with HbA1c of $\geq 7.5 \%$. However, monitoring of baseline data showed the actual proportion of participants with an $\mathrm{HbA1c}$ of $\geq 7.5 \%$ was around $90 \%$. A modelling exercise undertaken during recruitment with conservative estimates of $85 \%$ ( $\mathrm{HbA1c} \geq 7.5 \%$ ) and dropout rate of $15 \%$ suggested the trial would require at least 240 participants with primary outcome data at two years to preserve a power of at least $85 \%$.

\section{Randomisation}

After providing consent, participants were allocated to a training course, depending on their availability. Courses were randomised in pairs either to DAFNE plus pump or to DAFNE plus multiple daily injections. Simple randomisation in a block size of two, stratified by centre, was used for the first four courses. Courses 5 onwards were allocated in pairs using minimisation; the number of participants with baseline HbA1c values $\geq 7.5 \%$ and the total number of participants were used as minimisation 
factors. A statistician within Sheffield Clinical Trials Research Unit conducted the randomisation by a user written Stata code produced to generate allocation. The trial coordinator revealed the allocation to study sites. Participants who were unable to attend their original course were allowed to attend a later course in the same treatment arm, to reduce selection bias.

\section{Statistical analysis}

Analyses were performed in Stata 13 after a prespecified approved statistical analysis plan. All analyses were by intention to treat, with participants analysed in the groups to which they were randomised, unless otherwise specified. Participants were included in the intention to treat analysis if they had at least one post-baseline HbA1c measure. Those who dropped out before receiving the intervention were substituted where possible, to ensure the courses were run with adequate numbers of participants, but these individuals were not included in the analysis. Statistical tests were two sided at the $5 \%$ significance level.

We analysed the change in HbA1c (\%) at two years using a mixed effects model, with centre and baseline HbA1c treated as fixed effect covariates, and course (cluster) as a random intercept. For the primary analysis we used multiple imputation to impute missing $\mathrm{HbA1c}$ data for participants with at least one follow-up HbA1c measure but without two years outcome. We also performed a per protocol sensitivity analysis that excluded participants who had switched treatment. Changes in $\mathrm{HbA1c}$ values at six months and one year were analysed in the same way.

The proportion of participants reaching an HbA1c of $<7.5 \%$ was compared between groups using a logistic regression model adjusted for baseline $\mathrm{HbA1c}$, and centre and modelling separate courses within centre as random intercepts.

We used negative binomial mixed effects regression (to account for over-dispersion and clustering) on the number of moderate hypoglycaemic episodes in the four weeks before each follow-up, and occurrence of at least one moderate episode in the four weeks before courses as a covariate and the same covariates described previously. The number of severe hypoglycaemic episodes in two years was analysed as for moderate hypoglycaemic episodes, with the addition of study follow-up as the exposure. Incidence rate ratios were calculated using negative binomial random effects regression with participant as the random effect, adjusted for baseline HbA1c value and centre, based on the full intention to treat set $(n=260)$.

Secondary continuous outcomes (insulin dose, body weight, high density lipoprotein and total cholesterol levels) were analysed as for the primary outcome. We categorised proteinuria as macroalbuminuria, microalbuminuria, or normal and analysed using mixed effects ordered logistic regression adjusted for clustering by course (random effect), centre, and baseline HbA1c (fixed effects).

Changes in psychological outcomes were analysed using a mixed model adjusted for course (random), centre, baseline HbA1c, and baseline score, with the exception of the diabetes treatment satisfaction questionnaire, which we compared between groups using a non-parametric Wilcoxon-Mann-Whitney U test. No adjustments for multiple testing were made to the significance level for all exploratory secondary objectives.

A retrospective subgroup analysis used mixed effects regression modelling with the primary outcome, change in HbA1c (\%), including main effects of treatment group and subgroup, an interaction term between treatment and subgroup, and covariates of centre (fixed effect) and course (random effect). All categories for the subgroup analysis were prespecified in the statistical analysis plan but not in the original protocol, and are reported in full.

\section{Patient involvement}

Fifteen people, who had previously attended DAFNE courses (including pilot courses on pump treatment) but were not participating in the trial, were recruited to act as a user group and contribute to different aspects of the work. We invited two members to join both the steering group and the other investigator meetings. In addition, one of the project team (a doctor) is a pump user. They provided input to the trial design, implementation, and dissemination, including all participant materials. This included a discussion of the most appropriate research questions and whether individuals who were willing to try pump treatment could be successfully recruited into a trial where they could be randomised to multiple daily injections.

\section{Results}

\section{Study participants}

Participants were recruited between November 2011 and April 2013. Follow-up continued until June 2015. The CONSORT flowchart (fig 1) shows the flow of patients through the trial. Forty six courses were randomised. Of the 317 participants included in the randomisation, 50 were excluded from any analysis; 40 withdrew before giving baseline data and 10 before their course. All randomised courses were delivered. Of 267 participants randomised and attending the baseline assessment and the course, 260 (pump=132; injections=128) made up the intention to treat set. Two hundred and forty eight participants had complete primary outcome data at 24 months.

Table 1 shows the baseline demographics and characteristics of the trial population stratified by treatment received. Baseline data were well balanced between treatment groups, with the exception of slightly higher baseline HbA1c in the pump group (9.3\% v 9.0\%). Just $9 \%$ had a baseline HbA1c $<7.5 \%$.

\section{Primary outcomes}

HbA1c

At 24 months in participants whose baseline HbA1c was $\geq 7.5 \%$ ( $n=119$ in pump group; $n=116$ in multiple daily injections group) the mean change in the pump group was a decrease of $0.85 \%(9.3 \mathrm{mmol} / \mathrm{mol})$ 


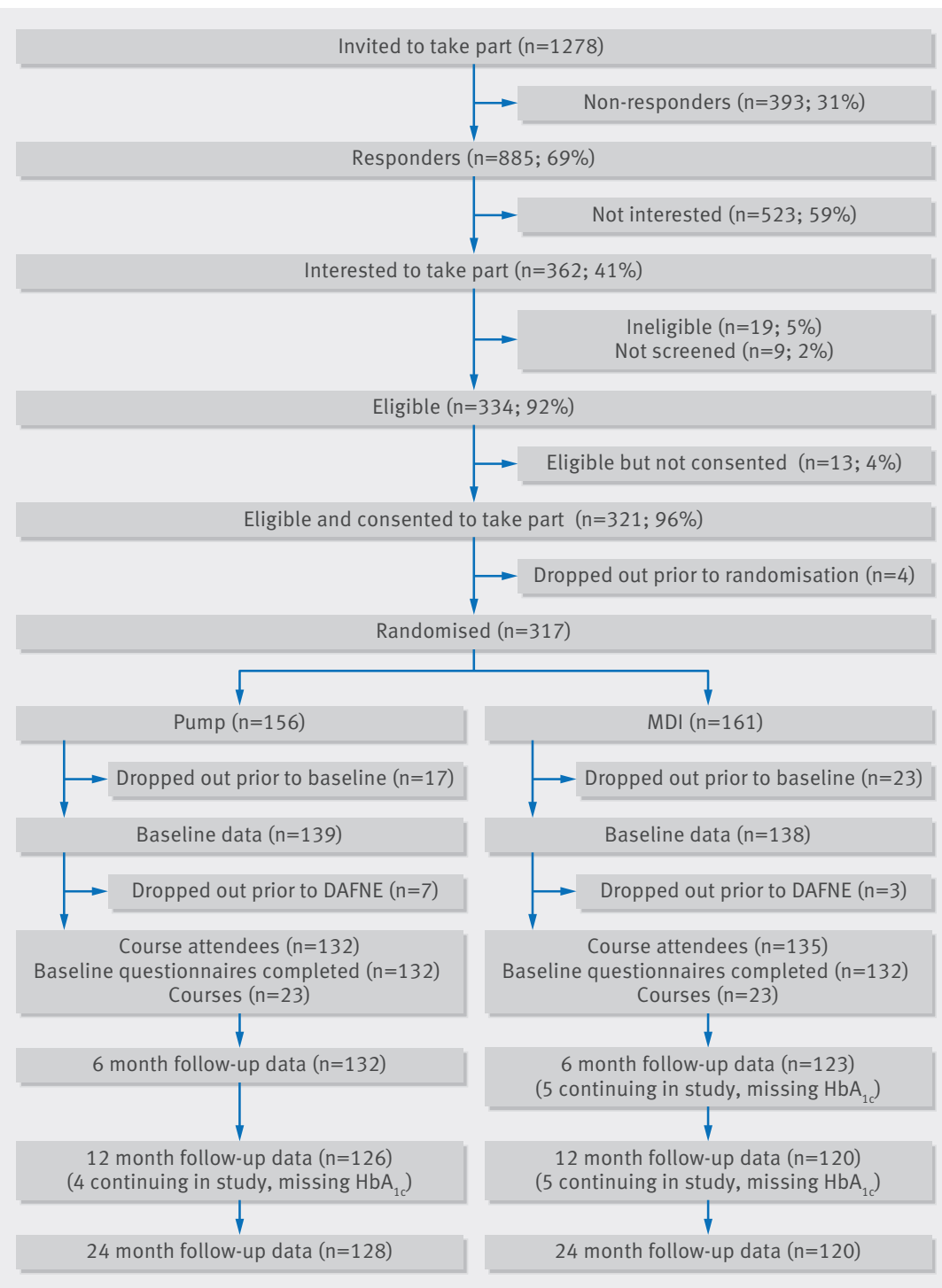

Fig 1 | CONSORT flowchart for REPOSE cluster randomised trial to compare the effectiveness of insulin pumps with multiple daily injections (MDI). DAFNE=dose adjustment for normal eating compared with $0.42 \%(4.5 \mathrm{mmol} / \mathrm{mol})$ in the multiple daily injections group. After adjusting for centre, course, and baseline HbA1c, the mean difference in HbA1c change from baseline was $-0.24 \%$ (95\% confidence interval $-0.53 \%$ to $0.05 \%)(-2.7 \mathrm{mmol} / \mathrm{mol},-5.8$ to 0.5$)$ in favour of pump treatment $(\mathrm{P}=0.10)$. The treatment difference was larger for the per protocol analysis; mean difference $-0.36 \%(-0.64 \%$ to $-0.07 \%)(-3.9$ $\mathrm{mmol} / \mathrm{mol},-7.0$ to -0.8 ) in favour of pump treatment $(\mathrm{P}=0.02)$, although this point estimate was still smaller than the prespecified minimal clinically important difference. Estimate of the intraclass correlation coefficient was approximately $0.5 \%$.

At 24 months, for the treatment groups combined $(\mathrm{n}=248)$, in all participants with complete HbA1c data there was a decrease of $0.54 \%$ ( $95 \%$ confidence interval $0.38 \%$ to $0.69 \%$ ) (5.9 mmol $/ \mathrm{mol}, 4.2$ to 7.6$)$ and for participants with baseline HbA1c $\geq 7.5 \% \quad(n=224)$ the decrease was slightly greater, at $0.64 \%$ (95\% confidence interval $0.48 \%$ to $0.80 \%$ ) (7 mmol/mol, 5.2 to 8.8 ).

\section{Proportion of participants reaching $\mathrm{HbA1C} \leq 7.5 \%$} (58 $\mathrm{mmol} / \mathrm{mol}$ )

The proportions of participants reaching an HbA1c $\leq 7.5 \%$ (58 $\mathrm{mmol} / \mathrm{mol}$ ) after two years, regardless of baseline $\mathrm{HbA} 1 \mathrm{c}$ value, were $25.0 \%$ for the pump group and $23.3 \%$ for the multiple daily injections group (odds ratio 1.22, 95\% confidence interval 0.62 to 2.39, $\mathrm{P}=0.57$, table 2 ). The results were similar at six and 12 months.

The primary analysis at 24 months was repeated for six and 12 month follow-up visits (table 3). The results for these interim time points were consistent with the primary outcome analysis. The largest difference in HbA1c change from baseline was observed at six months, with an adjusted mean difference of $-0.25 \%$ ( $95 \%$ confidence interval $-0.52 \%$ to $0.02 \%$ ) ( $-2.7 \mathrm{mmol} /$ mol, -5.6 to 0.2$), P=0.07$. Figure 2 displays the change in $\mathrm{HbA1c}$ for participants with data at all four time points by treatment group.

\begin{tabular}{|c|c|c|c|}
\hline Characteristics & $\begin{array}{l}\text { Pump treatment } \\
(n=132)\end{array}$ & $\begin{array}{l}\text { Multiple daily injections } \\
(\mathrm{n}=135)\end{array}$ & Total $(n=267)$ \\
\hline Mean (SD) age (years), range & 41.5 (14.2), 18.5-77.6 & 39.9 (12.5), $18.5-73.1$ & 40.7 (13.4), 18.5-77.6 \\
\hline Men & $78(59)$ & $82(61)$ & $160(60)$ \\
\hline White British & $125(95)$ & $119(88)$ & $244(91)$ \\
\hline Mean (SD) body mass index, range & $27.4(5.0), 17.4-47.9$ & $27.0(5.0), 17.2-45.9$ & $27.2(5.0), 17.2-47.9$ \\
\hline Mean (SD) duration of diabetes (years), range & $18.5(12.9), 1.1-56.9$ & $17.5(12.1), 1.1-51.9$ & $18.0(12.5), 1.1-56.9$ \\
\hline Any macrovascular complication & $68(52)$ & $79(59)$ & $147(55)$ \\
\hline Retinopathy & $51(39)$ & $65(48)$ & $116(43)$ \\
\hline Neuropathy & $13(10)$ & $6(4)$ & $19(7)$ \\
\hline \multicolumn{4}{|l|}{ Nephropathy: } \\
\hline Present & $26(20)$ & $24(18)$ & $50(19)$ \\
\hline Not calculable & $24(18)$ & $26(19)$ & $50(19)$ \\
\hline$\geq 1$ episodes of severe hypoglycaemia within past year & $16(12)$ & $15(11)$ & $31(12)$ \\
\hline $\mathrm{HbA1c} 7.5 \%$ & $119(90)$ & $123(91)$ & $242(91)$ \\
\hline Mean (SD) HbA1c (\%), range & $9.3(1.9), 5.7-16.7$ & $9.0(1.4), 6.1-14.1$ & 9.1(1.7), 5.7-16.7 \\
\hline Mean (SD) HbA1c (mmol/mol), range & 77.9 (21.0), 39.0-159.0 & $74.8(15.6), 43.0-131.0$ & 76.3 (18.5), 39.0-159.0 \\
\hline Total insulin dose (IU/weight) & $128(97)$ & $133(99)$ & $261(98)$ \\
\hline Mean (SD) total insulin dose (IU/weight), range & $0.72(0.28), 0.20-1.53$ & $0.75(0.29), 0.28-1.99$ & 0.74 (0.28), 0.20-1.99 \\
\hline
\end{tabular}




\begin{tabular}{|c|c|c|c|c|}
\hline Follow-up & $\begin{array}{l}\text { Pump treatment } \\
(\mathrm{n} / \mathrm{N}(\%))\end{array}$ & $\begin{array}{l}\text { Multiple daily } \\
\text { injections (n/N (\%)) }\end{array}$ & Odds ratio* $(95 \% \mathrm{Cl})$ & P value \\
\hline 6 months & $26 / 132(20.5)$ & $26 / 123(21.1)$ & 1.03 (0.51 to 2.10$)$ & 0.93 \\
\hline 12 months & $29 / 126(23.0)$ & $27 / 120(22.5)$ & 1.32 (0.62 to 2.80$)$ & 0.48 \\
\hline 24 months & $32 / 128(25.0)$ & $28 / 120(23.3)$ & 1.22 (0.62 to 2.39$)$ & 0.57 \\
\hline
\end{tabular}

Table 3 | Mean difference in change in HbA1c (\%) at six and 12 months in participants with baseline $\mathrm{HbA} 1 \mathrm{c} \geq 7.5 \%$

\begin{tabular}{|c|c|c|c|c|c|c|}
\hline \multirow[b]{2}{*}{ Follow-up } & \multicolumn{2}{|c|}{ Pump treatment } & \multicolumn{2}{|c|}{ Multiple daily injections } & \multirow{2}{*}{$\begin{array}{l}\text { Mean difference in } \\
\text { change* }(95 \% \mathrm{CI})\end{array}$} & \multirow[b]{2}{*}{$P$ value } \\
\hline & No & Mean (SD) change & No & Mean (SD) change & & \\
\hline 6 months & 118 & $-0.76(1.19)$ & 111 & $-0.36(1.06)$ & $-0.25(-0.52$ to 0.02$)$ & 0.07 \\
\hline 12 months & 111 & $-0.70(1.10)$ & 107 & $-0.40(1.02)$ & $-0.13(-0.40$ to 0.14$)$ & 0.35 \\
\hline
\end{tabular}

\section{Secondary outcomes Hypoglycaemia}

Relatively few severe hypoglycaemic episodes were observed post-baseline: 49 in 25 participants. The rate of severe hypoglycaemia during the 24 month follow-up did not differ between the treatment groups, adjusted for centre, course, baseline HbA1c, and presence of at least one severe hypoglycaemic episode in the 12 months before baseline (incidence rate ratio 1.13, 95\% confidence interval 0.51 to $2.51, \mathrm{P}=0.77$ ).

Across both treatment groups, the number of severe hypoglycaemic episodes was reduced. The average number of episodes for each patient per year in the study reduced from 0.17 before baseline to 0.10 during follow-up. The incidence rate ratio for the number of severe hypoglycaemic episodes in the 24 month follow-up, compared with the year before baseline, was 0.46 ( 0.24 to $0.89, \mathrm{P}=0.02$ ).

Across both treatment arms, on average, three moderate hypoglycaemic episodes were recorded for each patient over a four week history at six months. By 24 months this number was slightly lower (2.6 for pump group, 2.3 for multiple daily injections group) but there was no statistically significant difference between the groups in rates of moderate hypoglycaemic episodes at any time point.

\section{Other biomedical outcomes}

Body weight remained roughly constant throughout the study period, and was not statistically significantly

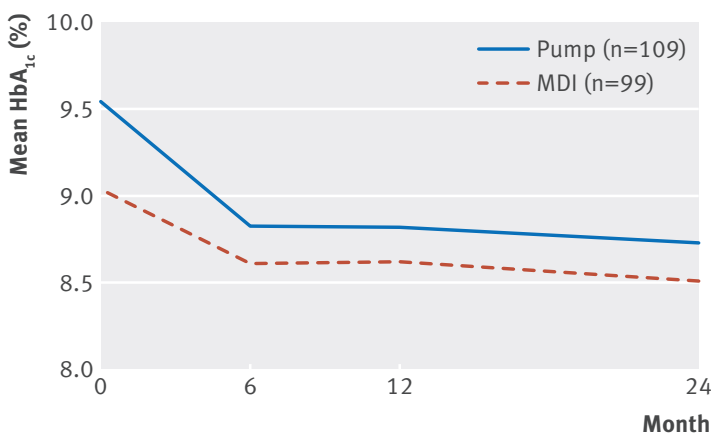

Fig 2 Mean change (\%) in glycated haemoglobin (HbA1c) over time in participants with baseline $\mathrm{HbA1c} \geq 7.5 \%$ (58 $\mathrm{mmol} / \mathrm{mol}$ ) (including only participants with data at all four time points, $n=208)$. MDI=multiple daily injections different between the treatment groups at any time point (table 4). A slight increase in high density lipoprotein cholesterol and a slight decrease in total cholesterol levels were observed in both groups, with no evidence of a difference between treatment groups in change from baseline ( $\mathrm{P}$ values ranged from 0.22 to 0.86). Insulin dose decreased in both arms. At 12 months, participants in the pump group had a $0.07 \mathrm{IU} /$ $\mathrm{kg}$ greater reduction in insulin dose than those in the multiple daily injections group (95\% confidence interval 0.01 to 0.13 decrease, $\mathrm{P}=0.02$ ). The difference was slightly smaller at six and 24 months and was not statistically significant. There was no statistically significant difference in the odds of proteinuria between the treatment groups at any time point (table 5).

\section{Diabetic ketoacidosis}

The number or type of serious adverse events did not differ between the groups, with the exception of diabetic ketoacidosis, which was greater in the pump group compared with multiple daily injections group (17 v5). More patients using pumps than using multiple daily injections had several episodes $(5 v 2)$ and the differences were confined to the first year, with four episodes in each group during the second year. Three episodes occurred in two participants who switched to pump treatment, and one in a participant who switched to multiple daily injections. Most episodes of ketoacidosis were caused by infections and $18 \%$ by set failure in those using pumps. Only five episodes occurred when participants implemented all sick day rules.

\section{Ancillary outcomes}

High levels of completion of the psychosocial questionnaires were observed across all questionnaires and time points (around $90 \%$ completed at each time point). The completion rate was slightly higher for participants allocated to the pump compared with multiple daily injections, which reflects the relative dropout rates in the two groups. No between group differences were found in the generic quality of life and health status instruments SF-12, WHOQOL-BREF, and EQ-5D, and the HADs score for depression and anxiety at six, 12, and 24 months (tables 6-8).

The overall diabetes specific quality of life (DSQOL) (on a 100 point scale) showed that both groups improved at 24 months, by a mean of 8.2 (SD 13.1) points in the pump group and 4.2 (SD 13.2) points in the multiple daily injections group. Both groups showed improvements in the DSQOL subdomains, which were greater in the pump group although not always reaching statistical significance.

The improvement in DSQOL diet restrictions was larger for the pump group compared with multiple daily injections group at both 12 and 24 months (12 month adjusted mean difference in change from baseline -4.1 (95\% confidence interval -7.2 to $-1.0, \mathrm{P}=0.01$ ); 24 month adjusted mean difference in change from baseline -5.1 (-8.6 to $-1.6, \mathrm{P}=0.004)$; lower scores represent better outcomes. The pump group also had greater improvement in DSQOL daily hassle or functions at both 12 and 


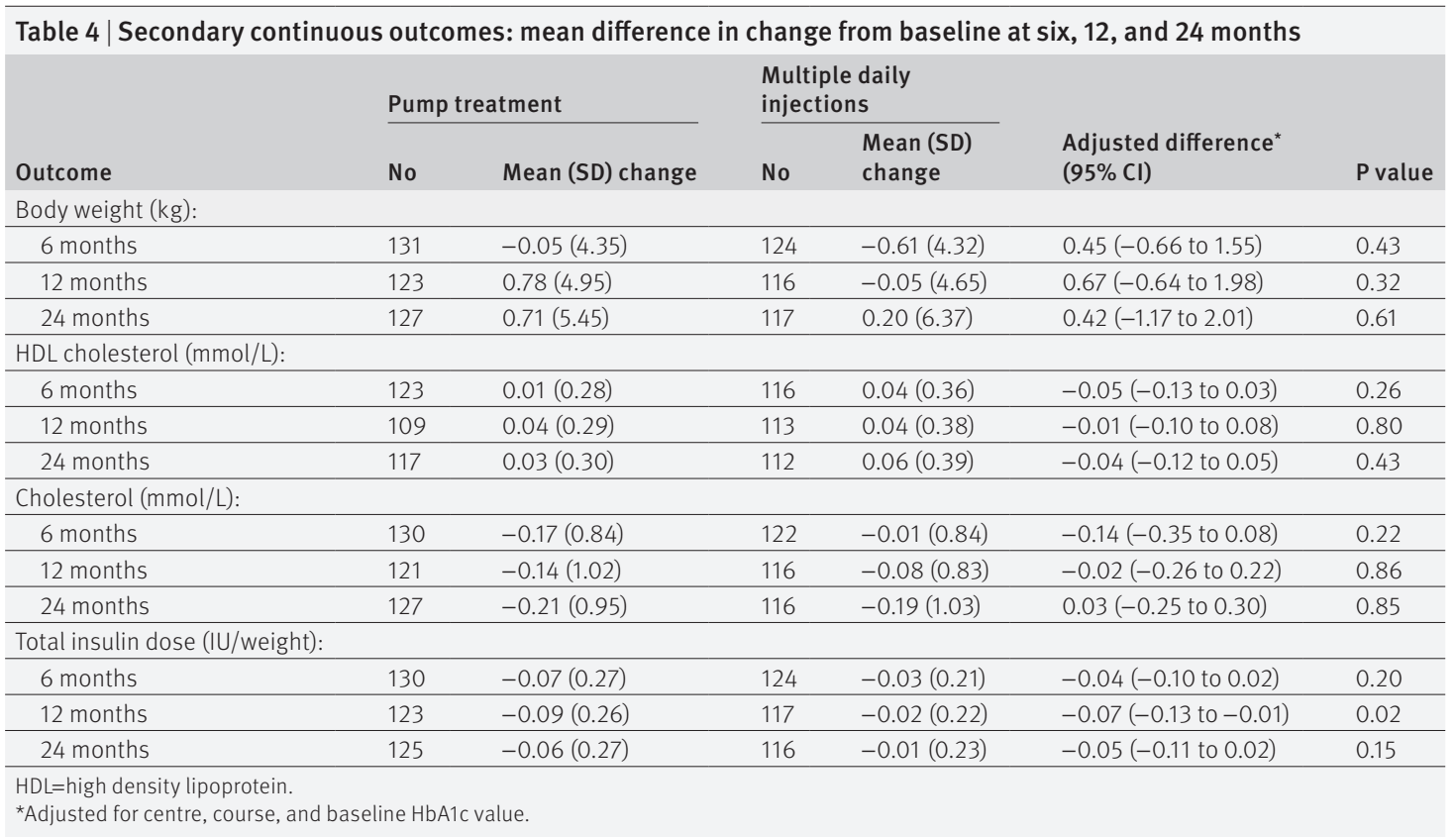

24 months; at 24 months the score had decreased by 10 points in the pump group, compared with 4 points in the multiple daily injections group (adjusted mean difference $-6.3,-10.9$ to $-1.8, \mathrm{P}=0.01$ ).

Participants in the pump group had better improvement in treatment satisfaction at all time points (table 9) but the difference was statistically significant at 12 and 24 months only $(\mathrm{P}=0.07$ at six months, $\mathrm{P}<0.001$ at 12 and 24 months).

\section{Retrospective analyses \\ Participants achieving the updated NICE recommendation of $\mathrm{HbA} 1 \mathrm{c} \leq 6.5 \%$}

A retrospective analysis showed that eight (3\%) of all participants (4/128 pump group and 4/120 multiple daily injections group) reached an $\mathrm{HbA} 1 \mathrm{c}$ of $\leq 6.5 \%$ (47 mmol/ mol) after two years (table 10). Of these, two participants (both in the pump group) experienced one or more episodes of severe hypoglycaemia during follow-up.

\begin{tabular}{|c|c|c|c|c|}
\hline Proteinuria category by follow-up & $\begin{array}{l}\text { Pump } \\
\text { treatment }\end{array}$ & $\begin{array}{l}\text { Multiple daily } \\
\text { injections }\end{array}$ & Odds ratio* $(95 \% \mathrm{Cl})$ & P value \\
\hline \multicolumn{5}{|l|}{6 months: } \\
\hline Normal & $76(80)$ & $81(81)$ & \multirow{3}{*}{0.79 (0.36 to 1.73 ) } & \multirow{3}{*}{0.56} \\
\hline Microalbuminuria & $17(18)$ & $14(14)$ & & \\
\hline Macroalbuminuria & $2(2)$ & $5(5)$ & & \\
\hline \multicolumn{5}{|l|}{12 months: } \\
\hline Normal & $65(76)$ & $67(81)$ & \multirow{3}{*}{1.14 (0.53 to 2.48$)$} & \multirow{3}{*}{0.74} \\
\hline Microalbuminuria & $16(19)$ & $10(12)$ & & \\
\hline Macroalbuminuria & $5(6)$ & $6(7)$ & & \\
\hline \multicolumn{5}{|l|}{24 months: } \\
\hline Normal & $77(81)$ & $70(83)$ & \multirow{3}{*}{1.04 (0.46 to 2.32) } & \multirow{3}{*}{0.93} \\
\hline Microalbuminuria & $16(17)$ & $9(11)$ & & \\
\hline Macroalbuminuria & $2(2)$ & $5(6)$ & & \\
\hline
\end{tabular}

*Adjusted for centre, course, and baseline HBA1c value.

\section{Participant contacts}

A retrospective analysis showed that, on average, those allocated to pumps had around double the number of contacts with professionals for diabetes between baseline and the end of year 1 , both face to face and by telephone. Between months 12 and 24, those using pumps had more face-to-face contacts, which were of longer duration (mean $1.6 v 1.3$ contacts), but fewer telephone contacts $(0.7 v 1.2)$.

\section{Blood glucose testing}

A retrospective analysis indicated that there was no difference in the mean frequency of blood glucose testing between treatment groups at 24 months after adjustment for baseline number of blood glucose tests, centre, and DAFNE course. The adjusted mean difference in blood glucose tests was 0.22 (95\% confidence interval -0.24 to 0.68 ) per day or 3.1 ( -3.4 to 9.6 ) over two weeks; $\mathrm{P}=0.35$. Overall, the number of blood glucose tests increased from 3.6 per day at baseline to 4.1 per day at 24 months (95\% confidence interval 0.33 to 0.82 , $\mathrm{P}<0.001)$.

\section{Subgroup analysis}

A retrospective analysis found no reliable statistical evidence of any subgroup effects or interactions between group (figs 3 and 4). However, there was some indication that participants with qualifications up to A level or equivalent did better in the pump group than in the multiple daily injections group (mean difference in HbA1c change (\%) at 24 months $-0.7 \%$ (95\% confidence interval $-1.2 \%$ to $-0.1 \%)(-7.4 \mathrm{mmol} / \mathrm{mol}, 95 \%$ confidence interval -13.2 to -1.5$)$ ), although the interaction test was not statistically significant $(\mathrm{P}=0.07)$.

\section{Discussion}

In a group of adults with type 1 diabetes referred for structured training in flexible insulin treatment because 


\begin{tabular}{|c|c|c|c|c|c|c|}
\hline \multirow[b]{2}{*}{ Outcomes } & \multicolumn{2}{|c|}{ Pump treatment } & \multicolumn{2}{|c|}{$\begin{array}{l}\text { Multiple daily } \\
\text { injections }\end{array}$} & \multirow[b]{2}{*}{$\begin{array}{l}\text { Adjusted difference* } \\
(95 \% \mathrm{Cl})\end{array}$} & \multirow[b]{2}{*}{$P$ value } \\
\hline & No & $\begin{array}{l}\text { Mean (SD) } \\
\text { change }\end{array}$ & No & $\begin{array}{l}\text { Mean (SD) } \\
\text { change }\end{array}$ & & \\
\hline SF-12 physical component summary ${ }^{\dagger}$ & 127 & $1.2(6.1)$ & 116 & $0.5(8.6)$ & $0.3(-1.4$ to 2.0$)$ & 0.72 \\
\hline SF-12 mental component summary & 127 & $0.2(8.8)$ & 117 & $0.9(9.9)$ & $-0.8(-2.9$ to 1.3$)$ & 0.45 \\
\hline \multicolumn{7}{|l|}{ DSQOL: } \\
\hline Total score $^{\dagger}$ & 128 & $-5.2(12.2)$ & 117 & $-4.4(11.2)$ & $-0.1(-2.8$ to 2.6$)$ & 0.94 \\
\hline Social relations & 128 & $-2.2(11.8)$ & 117 & $-3.0(13.2)$ & $1.5(-1.2$ to 4.2$)$ & 0.28 \\
\hline Leisure time restrictions and flexibility & 128 & $-5.1(16.6)$ & 117 & $-4.4(18.5)$ & $-0.1(-3.8$ to 3.7$)$ & 0.97 \\
\hline Physical complaints & 128 & $-6.0(17.0)$ & 117 & $-4.8(13.8)$ & $-0.1(-3.5$ to 3.3$)$ & 0.95 \\
\hline Worries about the future & 128 & $-7.9(20.4)$ & 117 & $-7.5(19.4)$ & $-0.7(-5.5$ to 4.1$)$ & 0.78 \\
\hline Daily hassle of functions & 128 & $-6.3(18.9)$ & 117 & $-5.0(18.7)$ & $-0.8(-5.0$ to 3.4$)$ & 0.70 \\
\hline Diet restrictions & 128 & $-11.3(18.3)$ & 117 & $-6.4(16.0)$ & $-3.3(-6.9$ to 0.2$)$ & 0.06 \\
\hline Treatment satisfaction (PWTSS) $^{\dagger}$ & 118 & $2.1(4.4)$ & 109 & $2.1(4.8)$ & $0.1(-0.7$ to 1.0$)$ & 0.79 \\
\hline \multicolumn{7}{|l|}{ WHOQOL-BREF: } \\
\hline Physical health $^{+}$ & 127 & $0.4(2.3)$ & 117 & $0.2(2.3)$ & $0.1(-0.4$ to 0.6$)$ & 0.74 \\
\hline Psychological & 128 & $0.1(1.9)$ & 117 & $0.4(2.2)$ & $-0.3(-0.7$ to 0.2$)$ & 0.23 \\
\hline Social relationships & 127 & $-0.3(2.7)$ & 117 & $0.3(3.0)$ & $-0.7(-1.3$ to 0.1$)$ & 0.03 \\
\hline Environment & 128 & $0.1(1.7)$ & 117 & $0.4(1.6)$ & $-0.3(-0.7$ to 0.1$)$ & 0.17 \\
\hline HFS behaviour score ${ }^{\ddagger}$ & 127 & $-1.7(4.9)$ & 117 & $-0.2(4.8)$ & $-0.9(-2.0$ to 0.1$)$ & 0.07 \\
\hline HFS worry score & 128 & $-4.0(10.9)$ & 117 & $-2.8(9.5)$ & $-0.1(-2.4$ to 2.1$)$ & 0.91 \\
\hline HADS anxiety scoren & 128 & $-0.2(3.0)$ & 117 & $-0.6(3.3)$ & $0.4(-0.3$ to 1.1$)$ & 0.26 \\
\hline HADS depression score & 128 & $-0.3(2.9)$ & 117 & $-0.2(2.5)$ & $0.1(-0.5$ to 0.7$)$ & 0.74 \\
\hline$E Q-5 D^{* *}$ & 127 & $-0.02(0.17)$ & 117 & $-0.01(0.18)$ & $-0.02(-0.06$ to 0.02$)$ & 0.38 \\
\hline \multicolumn{7}{|c|}{$\begin{array}{l}\text { SF12=12 item short form health survey; DSQOL=diabetes specific quality of life; PWTSS=preference weighted treatment satisfaction score; WHOQOL- } \\
\text { BREF=World Health Organization quality of life-BREF; HFS=hypoglycaemia fear survey; HADS=hospital anxiety and depression scale; EQ-5D=EuroQol } \\
\text { five dimensions questionnaire. } \\
\text { *Calculated using mixed effects regression adjusted for baseline quality of life score, centre, course, and baseline HBA1c. } \\
\text { †Scored from } 0 \text { (poor) to } 100 \text { (good). } \\
\text { fScored from } 10 \text { to } 50 \text { (higher score=greater fear). } \\
\text { §Scored from } 17 \text { to } 85 \text { (higher score=greater fear). } \\
\text { IScored from } 0 \text { (good) to } 21 \text { (poor). } \\
\text { **Scale from - } 0.56 \text { to } 1.00 \text { (good health). }\end{array}$} \\
\hline
\end{tabular}

of suboptimal diabetes control, participation in the REPOSE trial achieved a clinically worthwhile decrease in $\mathrm{HbA} 1 \mathrm{c}$ of $0.6 \%(7 \mathrm{mmol} / \mathrm{mol})$ at two years in those with a baseline HbA1c of $>7.5 \%$. However, in terms of the primary outcomes, there were no statistically significant differences in change from baseline to 24 months between those randomised to pump treatment or those using multiple daily injections, nor in the proportion of participants reaching an HbA1c of $\leq 7.5 \%$, indicating that pump treatment provided no clear biomedical benefit over training in DAFNE skills.

Rates of severe hypoglycaemia were halved in both groups (despite lower HbA1c values), a benefit maintained to 24 months with no difference between the groups in this or in rates of moderate hypoglycaemia. There were no other differences in biomedical outcomes apart from slightly greater reductions in insulin doses in those randomised to pump treatment. Both groups showed improved satisfaction with treatment and diabetes specific quality of life. Treatment satisfaction and two subdomains of the diabetes specific quality of life scale improved to a greater extent at two years in those allocated to pump treatment.

\section{Strengths and limitations of this study}

Compared with previous trials of pump treatment our study had a robust, multisite design, involved much larger numbers, and had a clinically meaningful period of follow-up pump treatment. Participants in both groups used analogue insulins and bolus calculators. The study was conducted in experienced secondary care centres and involved attendance at a structured education intervention that is well established across the UK. It included a comprehensive psychological evaluation with high levels of data completeness. The pragmatic study design thus provides good external validity, particularly as participating in the educational course led to sustained improvements in glycaemic control and reduced rates of severe hypoglycaemia.

It is not possible to blind a trial where insulin delivery systems are fundamentally different and this imposes limitations on any randomised controlled trial involving pumps. However, for the primary outcomes, HbA1c was objectively assessed using a central laboratory. ${ }^{17} \mathrm{~A}$ trial studying people who have expressed a desire for pump treatment is likely to struggle to recruit participants if one arm continues to use multiple daily injections. Those randomised to the injections arm might also either drop out or exhibit poor outcomes because of "disappointment" and lack of motivation. We recruited individuals who had not specifically requested pump treatment but were awaiting a course in diabetes self management to help them improve their metabolic control. Thus, our aim was to determine any added benefit of pumps over multiple daily injections while controlling for the training itself.

A potential limitation is that those randomised to pump treatment might have been insufficiently 


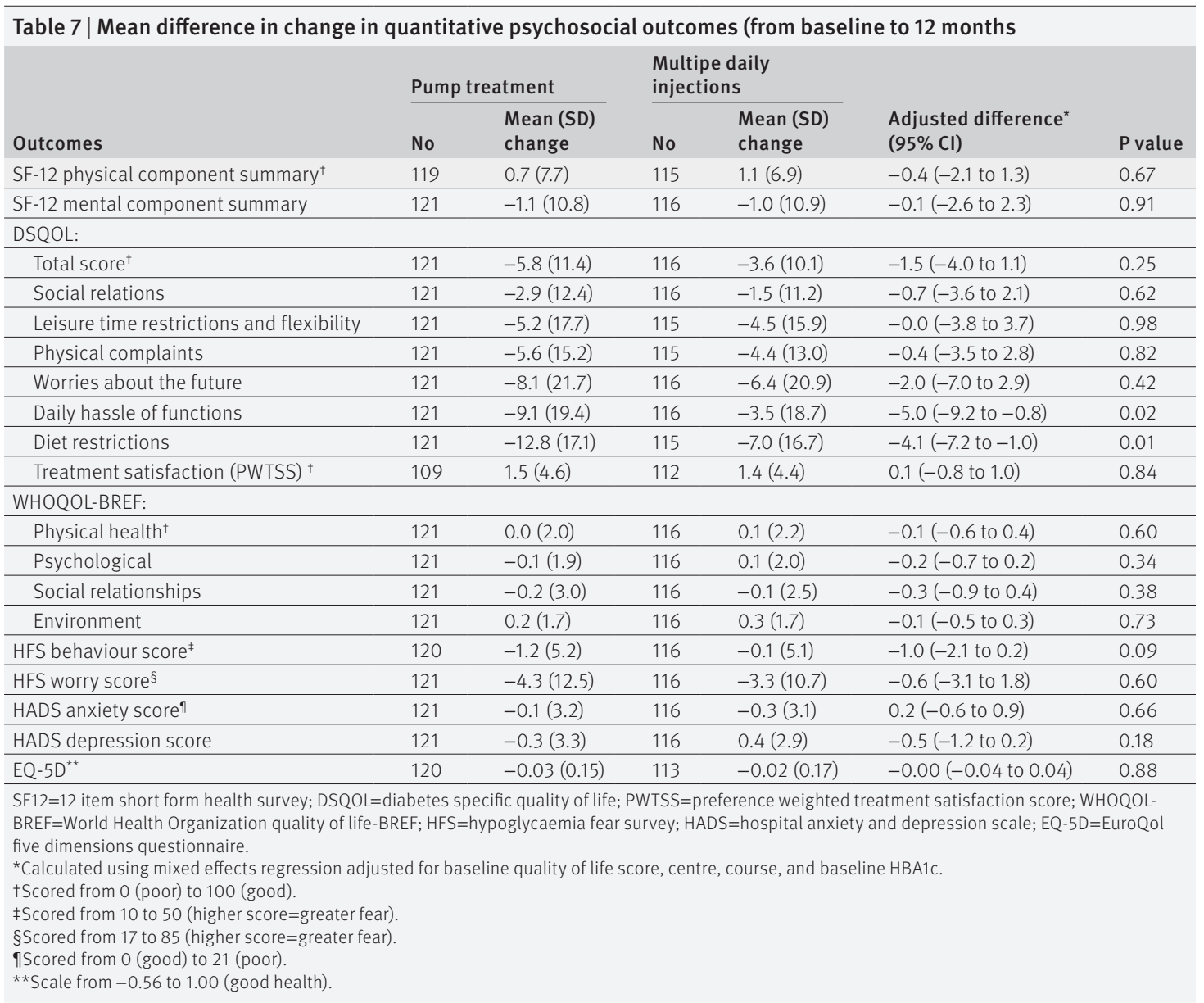

motivated to make the most of any technological benefit since they had not expressed a particular wish to use a pump. A common reason given by patients for not wanting to participate in REPOSE was reluctance to use an insulin pump. However, educators encouraged participants to use additional technological pump features (eg using more sophisticated bolus delivery) and provided extra input when this training was requested. Overall, we reasoned that since participants had signed up for a course to improve their glucose control, any added benefits of pump treatment would emerge.

In this pragmatic trial we did not collect detailed information about pump basal rates, how often patients adjusted and tested these, and time spent with pump participants. Our study was not designed to establish which features of pumps determine success in lowering HbA1c values in those doing "well” with pump treatment. An explanatory trial would have required a different study design. We therefore cannot be sure why those allocated to pump treatment failed to show a greater decrease in HbA1c.

We recorded the average number of blood glucose tests each day in both groups over the previous two weeks, both at baseline and at 24 months. Both groups had increased the number of daily tests from 3.6 before training to more than four each day at 24 months, but this did not differ between the groups. Perhaps this frequency of testing reflects a level of engagement in self management that was insufficient for participants in the pump group to take full advantage of the technology. One interpretation was that the educators were unable to provide adequate training in use of the pump during the one week course. However, it is just as likely that provision of pump treatment in a group of patients who had not been previously trained in delivery of flexible intensive insulin treatment included many who subsequently found it too challenging to implement and maintain the intensity of self management that both pump treatment and multiple daily injections demand.

\section{Comparison with other trials}

Two appraisals of pumps by NICE have reviewed the evidence on clinical and cost effectiveness. One ${ }^{18}$ noted that there were no trials of pumps against "best multiple daily injections" with long acting and short acting analogue insulins; that some trials had unequal amounts of education in the arms (with more in the pump arms); and that the trials had focused on easily measurable outcomes such as HbA1c, rather than on benefits in terms of flexibility of lifestyle and quality of life. The report recommended trials of pumps against analogue based multiple daily injections. A more recent report ${ }^{19}$ found only three trials in adults, one a pilot and the other involving 39 adults with type 1 diabetes, already using pump treatment who were randomised to continue with the pump or to switch to glargine based multiple daily injections. Patients received four months 


\begin{tabular}{|c|c|c|c|c|c|c|}
\hline \multirow[b]{2}{*}{ Outcomes } & \multicolumn{2}{|c|}{ Pump treatment } & \multicolumn{2}{|c|}{$\begin{array}{l}\text { Multiple daily } \\
\text { injections }\end{array}$} & \multirow[b]{2}{*}{$\begin{array}{l}\text { Adjusted difference* } \\
(95 \% \mathrm{Cl})\end{array}$} & \multirow[b]{2}{*}{$P$ value } \\
\hline & No & $\begin{array}{l}\text { Mean (SD) } \\
\text { change (SD) }\end{array}$ & No & $\begin{array}{l}\text { Mean (SD) } \\
\text { change }\end{array}$ & & \\
\hline SF-12 physical component summary ${ }^{\dagger}$ & 122 & $0.3(7.9)$ & 112 & $1.0(8.3)$ & $-0.4(-2.1$ to 1.3$)$ & 0.66 \\
\hline SF-12 mental component summary & 123 & $2.1(11.2)$ & 114 & $0.5(10.3)$ & $1.6(-0.7$ to 4.0$)$ & 0.18 \\
\hline \multicolumn{7}{|l|}{ DSQOL: } \\
\hline Total score $^{\dagger}$ & 123 & $-8.2(13.1)$ & 114 & $-4.2(13.2)$ & $-3.8(-6.5$ to -1.1$)$ & 0.01 \\
\hline Social relations & 123 & $-5.7(12.9)$ & 113 & $-2.7(14.8)$ & $-2.5(-5.4$ to 0.4$)$ & 0.09 \\
\hline Leisure time restrictions and flexibility & 123 & $-8.1(17.0)$ & 113 & $-3.6(19.7)$ & $-4.6(-8.4$ to -0.9$)$ & 0.02 \\
\hline Physical complaints & 123 & $-8.7(17.2)$ & 113 & $-4.8(16.6)$ & $-3.6(-7.3$ to -0.0$)$ & 0.05 \\
\hline Worries about the future & 123 & $-11.9(23.3)$ & 113 & $-7.8(21.2)$ & $-4.8(-9.7$ to 0.2$)$ & 0.06 \\
\hline Daily hassle of functions & 123 & $-9.6(21.2)$ & 113 & $-3.6(21.5)$ & $-6.3(-10.9$ to -1.8$)$ & 0.01 \\
\hline Diet restrictions & 123 & $-12.8(19.5)$ & 113 & $-6.9(19.3)$ & $-5.1(-8.6$ to -1.6$)$ & 0.004 \\
\hline 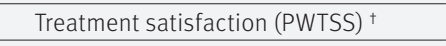 & 113 & $1.9(4.5)$ & 108 & $1.5(5.4)$ & $0.5(-0.5$ to 1.4$)$ & 0.32 \\
\hline \multicolumn{7}{|l|}{ WHOQOL-BREF: } \\
\hline Physical health $^{\dagger}$ & 123 & $0.5(2.4)$ & 114 & $-0.1(2.2)$ & $0.5(-0.0$ to 1.0$)$ & 0.07 \\
\hline Psychological & 123 & $0.5(2.5)$ & 114 & $0.3(2.4)$ & $0.2(-0.4$ to 0.7$)$ & 0.57 \\
\hline Social relationships & 123 & $0.0(3.3)$ & 114 & $0.1(2.9)$ & $-0.2(-0.9$ to 0.5$)$ & 0.63 \\
\hline Environment & 122 & $0.4(2.2)$ & 114 & $0.3(2.0)$ & $0.3(-0.2$ to 0.8$)$ & 0.21 \\
\hline HFS behaviour score ${ }^{\ddagger}$ & 122 & $-1.4(5.6)$ & 114 & $-0.6(5.1)$ & $-0.4(-1.5$ to 0.7$)$ & 0.44 \\
\hline HFS worry score ${ }^{\S}$ & 123 & $-6.7(13.0)$ & 114 & $-2.9(12.5)$ & $-3.4(-6.0$ to -0.8$)$ & 0.01 \\
\hline HADS anxiety score? & 123 & $-1.0(4.0)$ & 114 & $-0.5(3.5)$ & $-0.5(-1.3$ to 0.4$)$ & 0.26 \\
\hline HADS depression score & 123 & $-1.0(3.8)$ & 114 & $-0.2(3.3)$ & $-0.7(-1.5$ to 0.1$)$ & 0.11 \\
\hline$E Q-5 D^{* *}$ & 123 & $-0.00(0.18)$ & 113 & $-0.02(0.18)$ & $0.02(-0.03$ to 0.06$)$ & 0.46 \\
\hline \multicolumn{7}{|c|}{$\begin{array}{l}\text { SF12=12 item short form health survey; DSQOL=diabetes specific quality of life; PWTSS=preference weighted treatment satisfaction score; WHOQOL- } \\
\text { BREF=World Health Organization quality of life-BREF; HFS=hypoglycaemia fear survey; HADS=hospital anxiety and depression scale; EQ-5D=EuroQol } \\
\text { five dimensions questionnaire. } \\
\text { *Calculated using mixed effects regression adjusted for baseline quality of life score, centre, course, and baseline HBA1c. } \\
\text { †Scored from } 0 \text { (poor) to } 100 \text { (good). } \\
\text { †Scored from } 10 \text { to } 50 \text { (higher score=greater fear). } \\
\text { §Scored from } 17 \text { to } 85 \text { (higher score=greater fear). } \\
\text { IScored from } 0 \text { (good) to } 21 \text { (poor). } \\
\text { **Scale from - } 0.56 \text { to } 1.00 \text { (good health). }\end{array}$} \\
\hline
\end{tabular}

treatment with each. A third trial recruited 57 adults randomised to pump or analogue injections in an equivalence study. None showed any difference in HbA1c. Thus, the evidence base from trials for comparing pumps and "best multiple daily injections" was weak in terms of numbers, with a total of only 103 patients and short follow-up.

The assessment for the second appraisal ${ }^{19}$ reviewed observational studies of adults switching to pumps for clinical indications. These have the advantage of measuring change in glycaemic control and hypoglycaemia in those who have most to gain, and these studies showed improved HbA1c of the order of around $0.5 \%$. Bias in observational studies is more of a problem, and results must be treated with caution. Furthermore, of 48 observational studies, only nine reported quality of life. Study numbers were small, with at most 35 patients. Duration was usually short. The longest

\begin{tabular}{|c|c|c|c|c|c|c|}
\hline \multirow[b]{2}{*}{ Outcomes } & \multicolumn{2}{|c|}{ Pump treatment } & \multicolumn{2}{|c|}{ Multiple daily injections } & \multirow[b]{2}{*}{ Difference $^{*}(95 \% \mathrm{Cl})$} & \multirow[b]{2}{*}{ Pvalue } \\
\hline & No & Median (interquartile range) & No & Median (interquartile range) & & \\
\hline \multicolumn{7}{|l|}{6 months: } \\
\hline Perceived frequencyt of hyperglycaemia & 126 & $-1(-2$ to 0$)$ & 116 & $-1(-2$ to 1$)$ & $-0.0(-1.0$ to 0.0$)$ & 0.18 \\
\hline Perceived frequencyt of hypoglycaemia & 127 & $0(-1$ to 1$)$ & 116 & $-1(-2$ to 0$)$ & $0.0(-0.0$ to 1.0$)$ & 0.30 \\
\hline Treatment satisfaction (scores 0 to 36 ) $\ddagger$ & 126 & $8(3$ to 12$)$ & 116 & $5(1$ to 10$)$ & $2.0(1.0$ to 4.0$)$ & 0.07 \\
\hline \multicolumn{7}{|l|}{12 months: } \\
\hline Perceived change in frequency of hyperglycaemia & 121 & $0(-2$ to 1$)$ & 118 & 1 ( -1 to 2$)$ & $0.0(-0.0$ to 1.0$)$ & 0.13 \\
\hline Perceived change in frequency§ of hypoglycaemia & 121 & $-1(-2$ to 0$)$ & 118 & $-1(-2$ to 0$)$ & $0.0(-0.0$ to 1.0$)$ & 0.35 \\
\hline Treatment satisfaction (change) (scores -18 to 18$) \ddagger$ & 121 & 16 (13 to 18$)$ & 118 & $12(7$ to 16$)$ & $-3.0(-4.0$ to -1.0$)$ & $<0.001$ \\
\hline \multicolumn{7}{|l|}{24 months: } \\
\hline Perceived frequencyt of hyperglycaemia & 122 & $-1(-2$ to 0$)$ & 113 & $-1(-2$ to 0$)$ & $-0.0(-1.0$ to 0.0$)$ & 0.07 \\
\hline Perceived frequencyt of hypoglycaemia & 123 & $0(-1$ to 1$)$ & 113 & $0(-2$ to 0$)$ & 0.0 (-0.0 to 1.0) & 0.50 \\
\hline Treatment satisfaction $\neq$ & 122 & $8(3$ to 12$)$ & 113 & 5 (0 to 9$)$ & $4.0(2.0$ to 5.0$)$ & $<0.001$ \\
\hline
\end{tabular}

* $95 \%$ confidence interval for median difference calculated as described in Newson. ${ }^{16}$ Calculated using Wilcoxon-Mann-Whitney U test.

tScored from 0 (infrequent) to 6 (frequent).

FHigher scores represent better outcomes.

$\S S$ cored from -3 (less often) to 3 (more often). 


\begin{tabular}{|c|c|c|}
\hline Follow-up & $\begin{array}{l}\text { Pump treatment } \\
(\mathrm{n} / \mathrm{N}(\%))\end{array}$ & $\begin{array}{l}\text { Multiple daily } \\
\text { injections (n/N (\%)) }\end{array}$ \\
\hline 6 months & $3 / 132(2.3)$ & $2 / 123(1.6)$ \\
\hline 12 months & $3 / 126(2.4)$ & $3 / 120(2.5)$ \\
\hline 24 months & 4/128 (3.1) & $4 / 120(3.3)$ \\
\hline \multicolumn{3}{|c|}{$\begin{array}{l}\text { Number of participants achieving } \leq 6.5 \% \text { is small and hence statistical } \\
\text { models to estimate difference in proportions and confidence intervals } \\
\text { failed to converge. }\end{array}$} \\
\hline
\end{tabular}

study noted that initial benefits from pump treatment might not be sustained. The present study has thus addressed several of these concerns with large numbers in an adequately powered trial and a virtually complete dataset for both biomedical and psychological outcomes.

\section{Clinical and policy implications}

Our study suggests that extending the availability of pumps to adults with type 1diabetes with suboptimal glycaemic control and no desire to use this form of insulin delivery is unlikely to result in either lower levels of

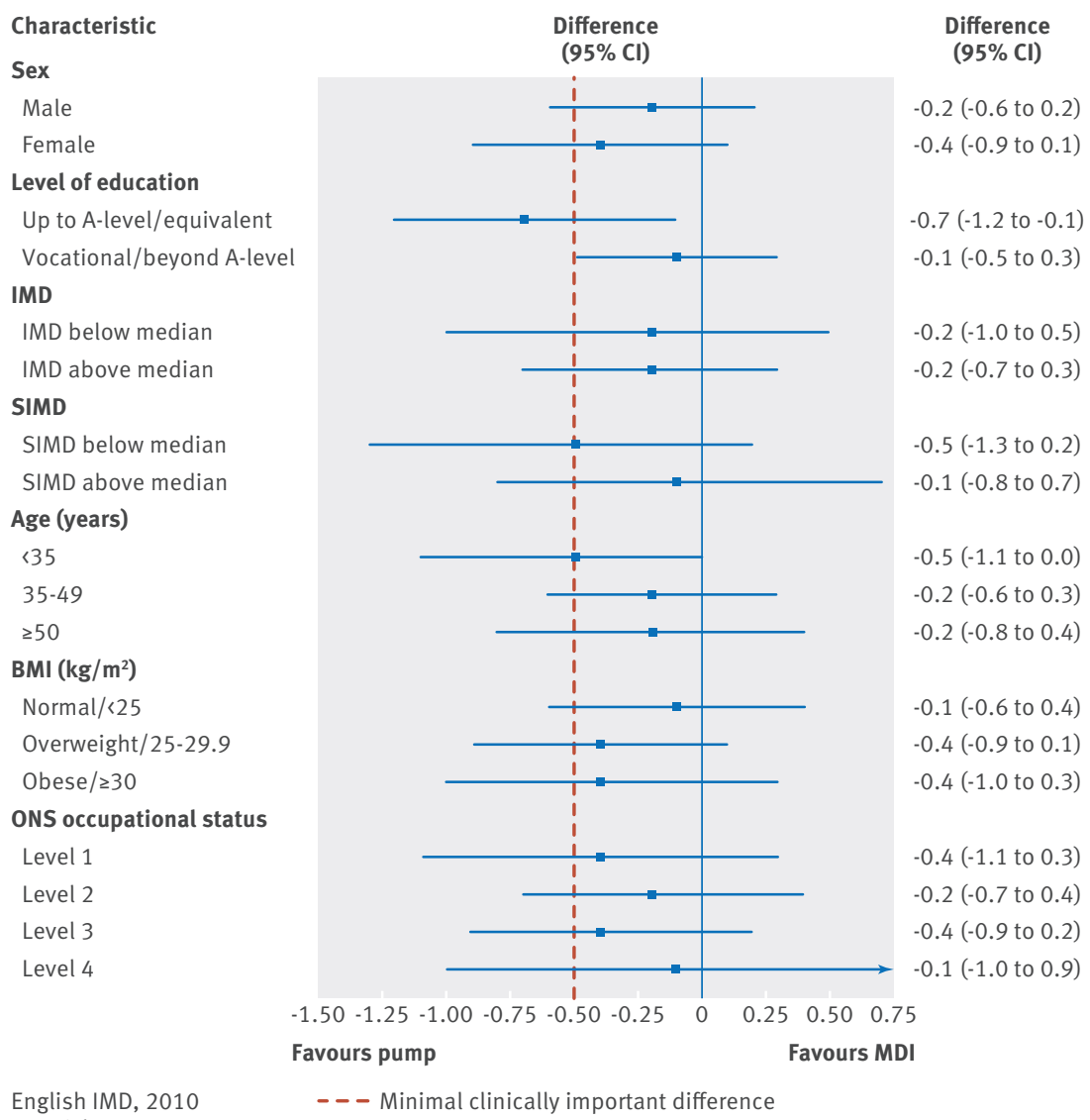

Scottish IMD, 2012

Fig 3 Mean difference in HbA1c change (\%) at 24 months for pump versus multiple daily injections by subgroup. $M C I D=$ minimal clinically important difference; IMD=index of multiple deprivation; ONS=Office for National Statistics occupational status from level 1 , elementary trade, service, administration roles, to level 4, corporate managers or directors, research, teaching, business, and public service higher level professionals glycaemia as measured by HbA1c or lower rates of hypoglycaemia. The absence of a control arm in which structured training was not provided, means we cannot be certain that participation in training explains the considerable decreases in HbA1c and severe hypoglycaemia in both groups. Yet, it seems unlikely that mere trial participation led to sustained decreases in HbA1c and severe hypoglycaemia for up to two years, particularly as allocation to such a control arm, in previous trials involving the DAFNE intervention, showed no effect on HbA1c. ${ }^{2}$

The results would appear to support the current clinical pathway as proposed by NICE, in which people desiring improved diabetes control undergo structured training in flexible insulin treatment with multiple daily injections alone. The trial outcomes, together with the review for NICE of observational pump studies, suggest that pumps might usefully be reserved to support those who actively engage in self management after structured education. Those who find that despite their best efforts, injections fail to deliver the expected benefits could then be offered the additional technological advantages of an insulin pump.

Clearly some patients improved more than others in terms of glucose control or hypoglycaemia and we explored whether there were any demographic differences in those who did particularly well. There was no reliable evidence of any plausible subgroup effects or interactions between the pump and multiple daily injections group, and the baseline characteristics of those whose glycaemic control changed to $<7.5 \%$ during the trial were no different from the pump population as a whole. We observed modest centre effects but no systematic differences according to greater experience in pump treatment.

Those using insulin pumps did show some quality of life benefits, reporting less restriction in diet and daily hassles in the DSQOL scale and greater treatment satisfaction. Nevertheless, the differences were modest and observed in comparison with a group given no novel technology. Since they were not associated with other positive treatment outcomes, those observations are probably insufficient to justify a major alteration in guidelines for the use of pumps.

One of the more striking results of this trial was the generally high level of HbA1c among adults in the UK enrolling for self management training in flexible insulin treatment. Participation in the courses produced important and sustained decreases, but for most participants still fell well short of the target recommended by NICE, recently reduced from $7.5 \%$ to $6.5 \% .{ }^{20}$ The high levels of HbA1c among people with type 1 diabetes in UK centres compared with most other European countries have also been noted in a recent study. ${ }^{21}$ There is an urgent need to explore the barriers to successful self management in adults with type 1 diabetes in the UK and to understand why referral for appropriate training is often left so long. This was also the conclusion of our recently completed research programme. ${ }^{15}$ Our results suggest that these problems cannot be overcome merely by providing additional technology in the form of pumps. 


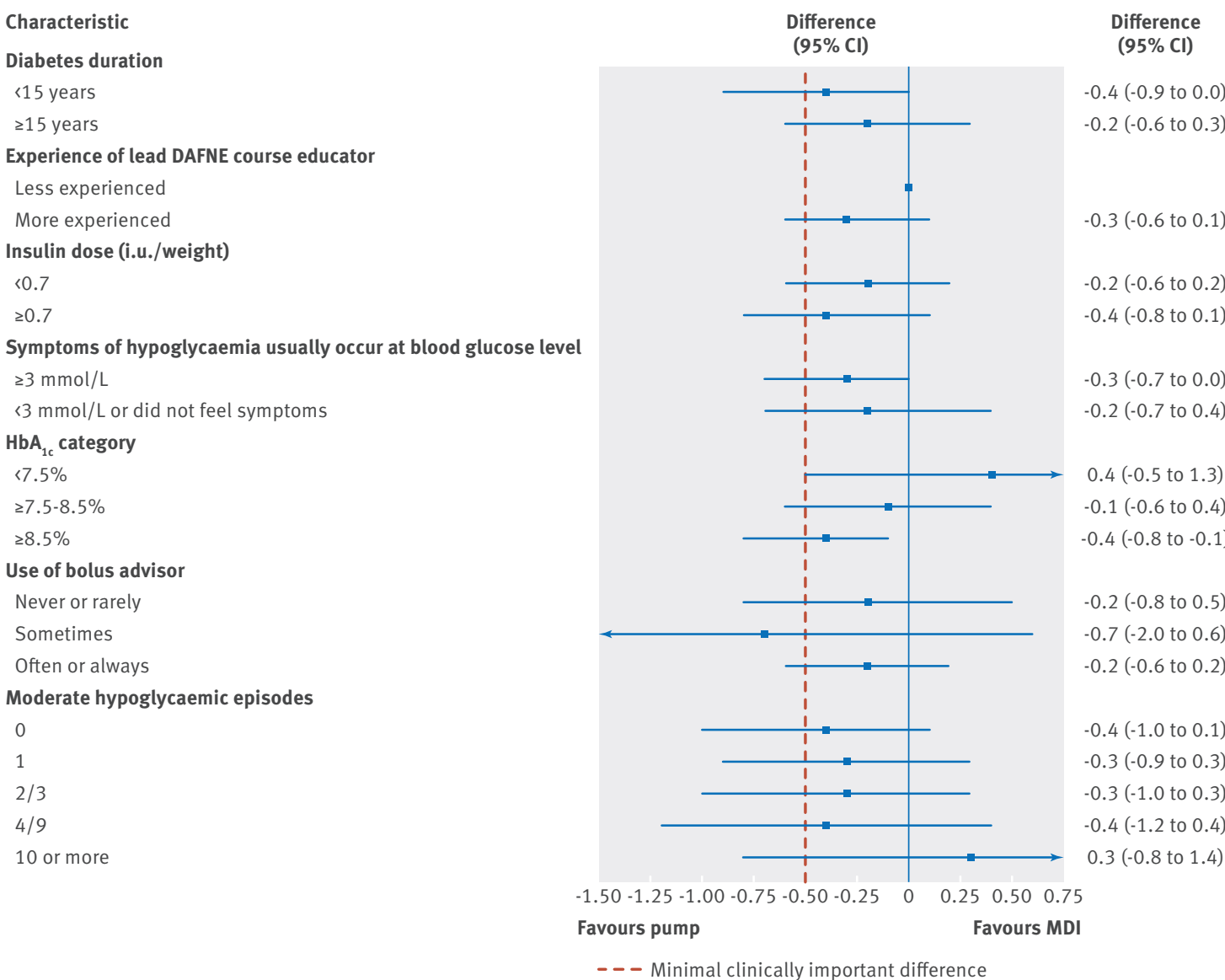

Fig 4 | Mean difference in HbA1c change (\%) at 24 months by subgroup

\section{Conclusions}

People with type 1 diabetes might be better served by ensuring far greater availability of high quality, structured self management training, which is currently only accessed by around $10 \%$ of adults in the UK. ${ }^{22}$ Participants might only recognise the limitations of insulin delivery by multiple daily injections if they start actively managing their diabetes after training. Those individuals could then be offered pump treatment to help them reach the stringent glucose targets necessary to achieve an $\mathrm{HbA1c}$ of $6.5 \%$ or to overcome problematic hypoglycaemia.

We thank those with diabetes who participated in the trial and members of the trial steering committee and data monitoring and ethics committee for their contribution. The research governance sponsor was Sheffield Teaching Hospitals NHS Foundation Trust. The clinical sites taking part were: Cambridge University Hospitals NHS Foundation Trust, Dumfries and Galloway Royal Infirmary, NHS Greater Glasgow and Clyde, Harrogate and District NHS Foundation Trust, King's College Hospital NHS Foundation Trust, NHS Lothian, Nottingham University Hospitals NHS Trust, and Sheffield Teaching Hospitals NHS Foundation Trust.

\section{Writing committee on behalf of the REPOSE Study Group}

Simon Heller (Department of Oncology and Metabolism, University of Sheffield), Stephanie Amiel (Kings College, University of London), Michael Campbell (School of Health and Related Research, ScHARR, University of Sheffield), Pratik Choudhary (Kings College, University of London), Cindy Cooper (Sheffield Clinical Trials Research Unit, University of Sheffield), Munya Dimairo (Sheffield Clinical Trials Research Unit, University of Sheffield), Jackie Elliott (Department of Oncology and Metabolism, University of Sheffield), Peter Hammond (Harrogate and District Foundation Trust), Ellen Lee (Sheffield Clinical
Trials Research Unit, University of Sheffield), Robert Lindsay (University of Glasgow), Peter Mansell (Nottingham University Hospitals NHS Trust), Norman Waugh (Warwick Medical School, University of Warwick) and David White (Sheffield Clinical Trials Research Unit, University of Sheffield).

\section{Members of the REPOSE Study Group}

Simon Heller was the chief investigator. Norman Waugh was the deputy chief investigator. Stephanie Amiel, Mark Evans, Fiona Green, Peter Hammond, Alan Jaap, Brian Kennon, Robert Lindsay, and Peter Mansell were site principal investigators and contributed to the study design and data interpretation.

The following contributed at sites to participant recruitment, intervention delivery, and data collection: Jane Baillie, Anita Beckwith, Helen Brown, Karen Callaby, Katy Davenport, Sarah Donald, Jackie Elliott, Leila Faghahati, Sara Hartnell, Allison Housden, Kalbir Kaur Pabla, Nicola Croxon, Sheena Macdonald, Muna Mohammed, Vicky Steel, Katy Valentine, Pamela Young, Ann Boal, Patsy Clerkin, Lynn Doran, Joanne Flynn, Emma Gibb, Hilary Peddie, Bernie Quinn, Helen Rogers, Janice Shephard, Janet Carling, Ann Collins, Laura Dinning, Christine Hare, Joyce Lodge, Sutapa Ray, Debora Brown, Jenny Farmer, Alison Cox, Chris Cheyette, Pratik Choudhary, Linda East, June Ellul, Katherine Hunt, Kimberley Shaw, Ben Stothard, Lucy Diamond, Lindsay Aniello, Debbie Anderson, Kathy Cockerell, Vida Heaney, Alison Hutchison, Nicola Zammitt, Gayna Babington, Gail Bird, Janet Evans, Tasso Gazis, Nicola Maude, Karen Nunnick, Dawn Spick, Laura Fenn, Carla Gianfrancesco, Valerie Gordon, Linda Greaves, Susan Hudson, Valerie Naylor, Chloe Nisbet, Carolin Taylor, Karen Towse, and Candice Ward.

Cindy Cooper, Gemma Hackney, Diana Papaioannou, Emma Whatley, and David White provided central trial management, oversight, and monitoring. Mike Bradburn, Michael Campbell, Munya Dimairo, and Ellen Lee contributed to the statistics. Hasan Basarir, Alan Brennan, Simon Dixon, and Daniel Pollard contributed to the health economics. Nina Hallowell, Jackie Kirkham, Julia Lawton, and David Rankin designed and undertook the qualitative work. Katharine Barnard led the quantitative psychosocial work. Timothy Chater and Kirsty Pemberton provided data 
management. Fiona Allsop and Lucy Carr provided central administration. Pamela Royle conducted literature searches and exploratory analyses. Gill Thompson and Sharon Walker provided central DAFNE support. Pauline Cowling conducted the fidelity assessment. Henry Smithson provided user representation on the management group.

Contributors: Simon Heller, Stephanie Amiel, Michael Campbell, Pratik Choudhary, Cindy Cooper, Munya Dimairo, Jackie Elliott, Peter Hammond, Ellen Lee, Robert Lindsay, Peter Mansell, Norman Waugh, and David White provided input into the study design, data interpretation, and drafting the final paper. All had full access to all of the data in the study and take responsibility for the integrity of the data and the accuracy of the data analysis. $\mathrm{SH}$ is the guarantor.

Funding: This research was funded by the UK Health Technology Assessment Programme (project No 08/107/01) and will be published in full in Health Technology Assessment. See the HTA programme website for further project information. (http://www.nets.nihr.ac.uk/). This report presents independent research commissioned by the National Institute for Health Research (NIHR). We also acknowledge financial support from the Research and Development Programmes of the Department of Health for England and the Scottish Health and Social Care Directorates who supported the costs of consumables, and of Medtronic UK, which provided the insulin pumps for the trial. These funders had no involvement in the design of the protocol; the collection, analysis, and interpretation of the data; the writing of this paper; or the decision to submit this article for publication. The views and opinions expressed herein are those of the authors and do not necessarily reflect those of the HTA, NIHR, NHS, the Department of Health, or Medtronic UK.

Competing interests: All members of the writing committee have completed the ICMJE uniform disclosure form at www.icmje.org/ coi_disclosure.pdf and declare: grant funding from the UK Health Technology Assessment Programme and financial support from Medtronic UK, for the submitted work; PC reports personal fees and non-financial support from Medtronic UK and personal fees from Roche, outside the submitted work. JE reports personal fees from Astra Zeneca, Eli Lily, Merck Sharpe Dohme, Novo Nordisk, Sanofi Aventis, and Takeda, and non-financial support from Eli Lilly, Novo Nordisk, and Sanofi, outside the submitted work. PH reports personal fees from Medtronic UK, Johnson and Johnson, Roche, Novo Nordisk, and Lilly, outside the submitted work. RL reports personal fees from Eli Lilly and Novo Nordisk, outside the submitted work. SH reports grants from Medtronic UK during the conduct of the study, and personal fees from Sanofi Aventis, Eli Lilly, Takeda, NovoNordisk, and Astra Zeneca, outside of the submitted work. His institution has received remuneration from Eli Lilly, Boeringher Ingelheim, NovoNordisk, Eli Lilly, and Takeda, outside the submitted work; he is currently chief investigator on an NIHR programme grant for applied research: RP-PG-0514-20013, the purpose of which is to develop more effective training programmes to improve self management of type 1 diabetes; no other relationships or activities that could appear to have influenced the submitted work.

Ethical approval: The protocol was approved by the Research Ethics Committee North West, Liverpool East (11/H1002/10). Each participating centre gave NHS Research and Development approval. The protocol received MHRA clinical trials authorisation. All participants provided written informed consent before taking part in the study.

Data sharing: Requests for patient level data and statistical code should be made to the corresponding author and will be considered by the REPOSE trial management group who, although specific consent for data sharing was not obtained, will release data on a case by case basis following the principles for sharing patient level data as described by Smith et al. ${ }^{23}$ The presented data do not contain any direct identifiers, we will minimise indirect identifiers and remove free text data, to minimise the risk of identification.

Transparency: The guarantor (SH) affirms that the manuscript is an honest, accurate, and transparent account of the study being reported; that no important aspects of the study have been omitted; and that any discrepancies from the study as planned have been explained.

This is an Open Access article distributed in accordance with the Creative Commons Attribution Non Commercial (CC BY-NC 4.0) license, which permits others to distribute, remix, adapt, build upon this work non-commercially, and license their derivative works on different terms, provided the original work is properly cited and the use is noncommercial. See: http://creativecommons.org/licenses/by-nc/4.0/.

1 The Diabetes Control and Complications Trial Research Group. The effect of intensive treatment of diabetes on the development and progression of long-term complications in insulin-dependent diabetes mellitus. N Engl/ Med 1993;329:977-86. doi:10.1056/ NEJM199309303291401.
2 DAFNE Study Group. Training in flexible, intensive insulin management to enable dietary freedom in people with type 1 diabetes: dose adjustment for normal eating (DAFNE) randomised controlled trial. BMJ 2002;325:746-9. doi:10.1136/bmj.325.7367.746.

3 Mühlhauser I, Bruckner I, Berger M, et al. Evaluation of an intensified insulin treatment and teaching programme as routine management of type 1 (insulin-dependent) diabetes. The Bucharest-Düsseldorf Study. Diabetologia 1987;30:681-90. doi:10.1007/BF00296989.

4 Nathan DM, Cleary PA, Backlund JY, et al. Diabetes Control and Complications Trial/Epidemiology of Diabetes Interventions and Complications (DCCT/EDIC) Study Research Group. Intensive diabetes treatment and cardiovascular disease in patients with type 1 diabetes. N Engl J Med 2005;353:2643-53. doi:10.1056/NEJMoa052187.

5 Tamborlane WV, Bonfig W, Boland E. Recent advances in treatment of youth with Type 1 diabetes: better care through technology. Diabet Med 2001;18:864-70. doi:10.1046/j.1464-5491.2001.00626.x.

6 Pickup J, Keen H. Continuous subcutaneous insulin infusion at 25 years: evidence base for the expanding use of insulin pump therapy in type 1 diabetes. Diabetes Care 2002;25:593-8. doi:10.2337/ diacare 25.3.593.

7 Riemsma R, Corro Ramos I, Birnie R, et al. Type 1 diabetes: Integrated sensor-augmented pump therapy systems for managing blood glucose levels (The MiniMed Paradigm Veo System and the Vibe and G4 PLATINUM CGM system), a systematic review and economic evaluation. Health Technol Assess 2016;20:v-xxxi. doi:10.3310/ hta20170.

8 National Institute for Health and Care Excellence (NICE). Continuous subcutaneous insulin infusion for the treatment of diabetes mellitus. NICE technology appraisal guidance 151. 2008. Available from: https://www.nice.org.uk/guidance/ta151

9 Diabetes UK. The United Kingdom Insulin Pump Audit- Service Level Data Online Source. 2013. Available from: https://jdrf.org.uk/ wp-content/uploads/2015/10/The_United_Kingdom_Insulin_Pump_ Audit_May_2013.pd

10 Pickup J. Insulin pumps. Int J Clin Pract Supp/ 2011;170:16-9. doi:10.1111/j.1742-1241.2010.02574.x.

11 Pickup JC. Are insulin pumps underutilized in type 1 diabetes? Yes. Diabetes Care 2006;29:1449-52. doi:10.2337/dc06-0011.

12 Leotta SAS, Altomare M, Carletti S, Fontana LPM. Intensive diabetes management in type 1 diabetic patients in poor glycemic control treated with insulin pump therapy or multiple daily injections: Two years follow-up. 4th Int ConfAdv Technol Treat Diabetes 2011.

13 White D, Waugh N, Elliott J, et al. REPOSE group. The Relative Effectiveness of Pumps Over MDI and Structured Education (REPOSE): study protocol for a cluster randomised controlled trial. BMJ Open 2014;4:e006204. doi:10.1136/bmjopen-2014-006204.

14 Ziegler R, Cavan DA,, Cranston I, et al. Use of an insulin bolus advisor improves glycemic control in multiple daily insulin injection (MDI) therapy patients with suboptimal glycemic control: first results from the ABACUS trial.DiabetesCare; 2013;36:3613-9doi:10.2337/ dc13-0251.

15 Heller S, Lawton J, Amiel S, et al. Improving management of type 1 diabetes in the UK: the DAFNE programme as a research test-bed. A mixed-method analysis of the barriers to and facilitators of successful diabetes self-management, a health economic analysis, a cluster RCT of different model. Prog Grants Appl Res 2014;2:5.

16 Newson R. Confidence intervals for rank statistics: Percentile slopes, differences, and ratios. Stata J 2006;6:497-520.

17 Kahan BC, Cro S, Doré C), et al. Reducing bias in open-label trials where blinded outcome assessment is not feasible: strategies from two randomised trials. Trials 2014;15:456. doi:10.1186/1745-6215-15-456

18 Colquitt JL, Green C, Sidhu MK, Hartwell D, Waugh N. Clinical and cost-effectiveness of continuous subcutaneous insulin infusion for diabetes. Health Technol Assess 2004;8:iii, 1-171. doi:10.3310/ hta8430.

19 Cummins E, Royle P, Snaith A, et al. Clinical effectiveness and cost-effectiveness of continuous subcutaneous insulin infusion for diabetes: systematic review and economic evaluation. Health Technol Assess 2010;14:iii-iv, xi-xvi, 1-181. doi:10.3310/hta14110.

20 National Institute for Health and Care Excellence (NICE). Type 1 diabetes in adults: diagnosis and management. Clinical guideline NG17 2015. www.nice.org.uk/guidance/ng17/evidence/ full-guideline-435400241

21 McKnight JA, Wild SH, Lamb MJ, et al. Glycaemic control of Type diabetes in clinical practice early in the 21 st century: an international comparison. Diabet Med 2015;32:1036-50. doi:10.1111/dme.12676

22 Health and Social Care Information Centre. National Diabetes Audit 2012-2013 Report 1: care processes and treatment targets. Health and Social Care Information Centre website. 2014. www.hscic.gov.uk/ catalogue/PUB14970

23 Smith C, Hopkins C, Sydes M, et al. Good practice principles for sharing individual participant data from publicly funded clinical trials. Trials 2015;16:01doi:10.1186/1745-6215-16-S2-01. 